\title{
ORGANIZACIÓN DEL CAMPUS VIRTUAL DE LA UNIVERSIDAD DE LAS PALMAS DE GRAN CANARIA
}

\author{
(ORGANIZATION OF THE VIRTUAL CAMPUS OF THE UNIVERSITY OF LAS PALMAS DE GRAN \\ CANARIA)
}

José Juan Castro Sánchez

Universidad de Las Palmas de Gran Canaria (España)

\section{RESUMEN}

El Campus Virtual de la Universidad de Las Palmas de Gran Canaria está estructurado en 3 grandes instalaciones: Teleformación, Enseñanza Presencial y Trabajo Colaborativo. En la instalación de Teleformación se presta servicios a todos los estudios en modalidad no presencial. En estos momentos hay en marcha 5 títulos oficiales: Psicopedagogía, Magisterio, Turismo, Relaciones Laborales y Trabajo Social. En la instalación de Enseñanza Presencial están dadas de alta todas las asignaturas que componen la totalidad de las titulaciones presenciales de la ULPGC. Tras finalizar el curso pasado más de la mitad de los docentes (cerca de 800) habían utilizado el Campus para implementar una o varias de sus asignaturas. De la misma manera, más de 13.000 estudiantes habían hecho un uso activo del Campus Virtual. La instalación de Trabajo Colaborativo no tiene un uso docente sino que funciona como un entorno colaborativo virtual.

Palabras clave: teleformación, enseñanza apoyada en TIC, trabajo colaborativo a través de TIC.

\begin{abstract}
The Virtual Campus of the University of Las Palmas de Gran Canaria is structured in 3 great facilities: e-Learning, b-Learning and Cooperative Work. In installation of e-Learning gives services to all the studies in modality e-learning. In these moments have 5 official qualifications: Psycopedagogy, Teaching, Tourism, Labour Relations and Social Work. In the installation of b-Learning are given of discharge all the matter that compose the totality of the qualifications attend them of the ULPGC. After the course passed more than the half of the teachers (near 800) they had used the Virtual Campus to implement one or several of his matter. Of the same way more than 13.000 students had done an active use of the Virtual Campus. The installation of Cooperative Work does not have an educational use but it works as an environment cooperative virtually.
\end{abstract}


Key words: e-learning, b-learning, TIC supported cooperative work.

Las sugerencias de muchos maestros en ejercicio deseosos de cursar la titulación de Licenciado en Psicopedagogía, los estudiantes que no podían acceder a la enseñanza presencial por el límite de plazas, los informes técnicos pertinentes y las demandas de los Cabildos de las islas, impulsaron que la Universidad de Las Palmas de Gran Canaria decidiera poner en marcha la primera titulación a través de Internet a partir del curso 2001/2002.

Así, está Universidad se embarcó en la aventura de la Teleformación a través de la Licenciatura en Psicopedagogía, convirtiéndose en una de las pocas universidades españolas tradicionales en su enseñanza presencial, con una titulación oficial que puede ser cursada a través de las TIC.

En un principio la Licenciatura en Psicopedagogía en Línea estaba catalogada como una forma distinta de impartir la ya existente Licenciatura en Psicopedagogía presencial y, que a todos los efectos, pertenecía a la Facultad de Formación del Profesorado. Pero, a partir del año 2003 y a raíz de los cambios reflejados en la Ley Orgánica de Universidades (LOU), se crea la Estructura Teleformación ULPGC aprobada en el Consejo de Gobierno de 25 de Junio de 2003, en sesión ordinaria. Dicha Estructura se hizo cargo desde entonces de la Licenciatura en Psicopedagogía en modalidad no presencial.

Como culminación de este proceso de desarrollo, el 1 de abril de 2005 el Consejo de Gobierno aprobó el Reglamento de la Estructura Teleformación ULPGC modificado parcialmente en febrero de 2007 (Castro, 2007).

\section{TELEFORMACIÓN}

A través de Teleformación se articula una oferta educativa dirigida a los estudiantes que no pueden cursar la enseñanza presencial en nuestra universidad, mediante el uso de las TIC. Asimismo, pretende acercar la universidad a los ciudadanos interesados en realizar unos estudios universitarios, especialmente de otras islas e iniciar un camino hacia la superación de las barreras de la comunicación en un territorio discontinuo como el nuestro. 


\section{Oferta de estudios en modalidad no presencial a través de teleformación}

Desde que se iniciaron los estudios de Psicopedagogía a través de teleformación, la oferta de otros estudios en modalidad no presencial no ha dejado de incrementarse curso tras curso. A la titulación de Psicopedagogía se sumaron en el curso 20052006 las titulaciones de Turismo y Magisterio y un curso más tarde las de Relaciones Laborales y Trabajo Social. Paralelamente se ha ido incrementado la oferta de estudios de posgrados hasta tal punto que hoy en día son más los posgrados que se ofertan en modalidad no presencial que en modalidad presencial. De la misma manera, la oferta de cursos de Extensión Universitaria y de Formación Continua también se ha ido incrementando año tras año.

A continuación figura la oferta de estudios en modalidad no presencial a través de teleformación del último curso (2006-2007):

TITULACIONES OFICIALES DE GRADO: Maestro-Especialidad Educación Primaria, Psicopedagogía, Relaciones Laborales, Trabajo social y Turismo.

LIBRE CONFIGURACIÓN: 100 asignaturas.

DOCTORADOS: Formación del Profesorado, Gestión en la Nueva Economía, Procesos, Dinámicas y Diagnósticos Territoriales en Áreas Insulares y Sistemas Inteligentes y Aplicaciones Numéricas en la Ingeniería.

TÍTULOS PROPIOS DE GRADO: Diploma de especialista en Protocolo y Relaciones Institucionales.

TÍTULOS PROPIOS DE POSGRADO: III Master en Administración y Dirección de Empresas, Master en Calidad, Gestión Medioambiental y Prevención de Riesgos Laborales, Master en Dirección de Recursos Humanos y Administración del Personal, Master en Ingeniería de Confiabilidad y Riesgo, Master en Prevención de Riesgos Laborales, II Master en Protocolo, Comunicación y Relaciones Externas, IV Master en Procesos Educativos, Experto en Asesoría Laboral de Empresa, Experto en Contabilidad Financiera, Experto en Derecho de Empresa, Experto en Diseño, Implementación y Desarrollo de Acciones Formativas a través de TIC, Experto en Evaluación de la Calidad en la Enseñanza Superior, Experto en Gestión de la Calidad, Experto en Gestión de la Formación Ocupacional y Continua, Experto en Gestión Medioambiental, Experto en Inclusión y Diversidad, Experto en Tabaquismo, II 
Experto en Administración y Dirección de Empresas, II Experto en Alta Dirección Universitaria, II Experto en Dirección de Recursos Humanos, II Experto en Dirección Financiera, II Experto en Formación de Gestores de Entidades y Proyectos de Voluntariado, II Experto en Gestión de la I+D+I, II Experto en Obesidad, III Experto de Identificación e Intervención Educativa y Familiar con el Alumnado de Altas Capacidades Intelectuales, IV Experto de Atención a las Necesidades Educativas Especiales, IV Experto de Fundamentos Psicológicos, IV Experto de Orientación Educativa, IV Experto de Resolución de Conflictos en el Aula, IV Experto en Gestión Hotelera: Aplicaciones de la Innovación, la Calidad y la Sostenibilidad; y IX Experto en Prevención de Riesgos Laborales.

FORMACIÓN CONTINUA: Transferencia de la Tecnología, Outsourcing, Derecho Laboral, Habilidades Comunicativas, Resolución de Conflictos, Aprender a Negociar, Liderazgo eficaz, Autoempleo, Dirección de Reuniones y toma de Decisión, Salud Laboral en la Escuela, Formación y Orientación Laboral, Formación Específica para Empresas, Aprender a Trabajar en Equipo, Inteligencia emocional en las relaciones interpersonales, Estrategias de gestión del tiempo y planificación del trabajo, Conceptos claves del contexto laboral, Búsqueda de empleo, Emprendiduría, Planificación, control y gestión eficaz del presupuesto, Entornos virtuales de aprendizaje.

EXTENSIÓN UNIVERSITARIA: Programación en C SHARP, Desarrollo Web: PHP y Mysql, Introducción a linux, English for the technical environment, Enseñanza del inglés para fines específicos en la sociedad del conocimiento, Liderazgo eficaz de los equipos de trabajo, Formación y gestión de un equipo de ventas: aspectos psicológicos, Dirección de reuniones y toma de decisión, Estrategias de gestión del tiempo y planificación del trabajo, Recursos didácticos para la enseñanza de la estadística, Pérdida y duelo, Búsqueda activa de empleo, Manejo del estrés laboral, Manejointeligentedelas emociones, Trabajo en equipoymotivación, Enriquecimiento personal: los moldes de la mente, Técnicas de estudio, Autoempleo, Conceptos claves del contexto laboral, Inteligencia emocional en las relaciones interpersonales, Aprender a trabajar en equipo, Habilidades comunicativas, Resolución de conflictos, Aprender a negociar.

OTROS ESTUDIOS: Formación del Personal de Administración y Servicios de la ULPGC, Formación del Profesorado de la ULPGC, Cursos de Nivelación de Conocimientos para estudiantes de nuevo ingreso, Perfeccionamiento del Profesorado no universitarios, y Habilitación para el Cuerpo de Maestros. 
Para el próximo curso está previsto que se inicie el Título Propio Seguridad y Emergencias que cuenta con el apoyo y reconocimiento de la Dirección General de Seguridad y Emergencias, la Academia Canaria de Seguridad, Empresa Pública de Gestión de la Salud y Seguridad en Canarias, el Ayuntamiento de Adeje, etc. cuyo plan de estudios y adscripción a la Estructura de Teleformación fue aprobado en el Consejo de Gobierno del 24 de noviembre de 2006.

\section{Perfil y opiniones de los estudiantes}

A continuación mostramos la distribución de los 913 estudiantes matriculados en el curso 2006/2007 en función del lugar de residencia habitual y la titulación. También podemos ver los resultados académicos de los cursos anteriores y los resultados de la última encuesta de valoración aplicada a finales del curso 20052006.

\section{Distribución por lugar de residencia}

La siguiente tabla muestra a los estudiantes distribuidos por el lugar de residencia habitual. La mayoría reside en Gran Canaria, casi el $80 \%$, le sigue Lanzarote y a continuación Fuerteventura y Tenerife. En el conjunto del archipiélago reside más del 97\%. El resto reside en la Península o en el extranjero en las siguientes ciudades: Barcelona, Madrid, Badajoz, Murcia, Alicante, Almería, Baleares, Burgos, Málaga, Sevilla, Tarragona, Valencia y East Sussex (Reino Unido) (Ver Tabla 1).

\begin{tabular}{|c|c|c|c|c|c|c|c|}
\hline & $\begin{array}{c}\text { Gran } \\
\text { Canaria }\end{array}$ & \multicolumn{4}{|c|}{ Resto de islas } & \multicolumn{2}{|c|}{ Fuera de Canarias } \\
\hline \multirow{2}{*}{$\%$} & \multirow{2}{*}{79,4} & \multicolumn{4}{|l|}{18,1} & \multicolumn{2}{|l|}{2,5} \\
\hline & & 6,9 & 4,9 & 4,9 & 1,3 & 2,3 & 0,2 \\
\hline $\mathrm{N}$ & 725 & 63 & 45 & 45 & 12 & 21 & 2 \\
\hline & $\begin{array}{c}\text { Gran } \\
\text { Canaria }\end{array}$ & Lanzarote & Fuerteventura & Tenerife & $\begin{array}{c}\text { El Hierro y La } \\
\text { Palma }\end{array}$ & Península & Extranjero \\
\hline
\end{tabular}

Tabla 1. Distribución de estudiantes según lugar de residencia

Distribución por titulación

En la siguiente tabla mostramos la distribución por titulación de los 913 estudiantes matriculados en la Estructura Teleformación ULPGC. Destacan los 
estudiantes de Magisterio que con tan solo dos cursos ya son los más numerosos. Por el contrario, los estudiantes de Turismo son los menos numerosos a pesar de haber empezado el mismo año que magisterio. Los porcentajes de Relaciones Laborales y Trabajo Social corresponden a la única promoción matricula hasta el momento (Ver Tabla 2).

\begin{tabular}{|c|l|l|l|l|l|}
\cline { 2 - 6 } \multicolumn{1}{c|}{} & \multicolumn{5}{c|}{ TITULACIONES } \\
\hline TOTAL & Psicopedagogía & \multicolumn{1}{c|}{ Turismo } & Magisterio & $\begin{array}{c}\text { Relaciones } \\
\text { Laborales }\end{array}$ & Trabajo Social \\
\hline 913 & 217 & 124 & 311 & 144 & 117 \\
$100 \%$ & $23,8 \%$ & $13,6 \%$ & $34,1 \%$ & $15,8 \%$ & $12,8 \%$ \\
\hline
\end{tabular}

Tabla 2. Distribución de estudiantes según titulación

\section{Resultados académicos}

En las siguientes tablas figuran los resultados académicos de los cursos anteriores ya finalizados. Los datos representan el porcentaje de aprobados sobre presentados y sólo hacen referencia a las titulaciones de Psicopedagogía, Magisterio y Turismo, las únicas que ya han finalizado al menos un curso (Ver Tablas 3.1, 3.2 y 3.3).

\begin{tabular}{|c|c|c|c|c|c|}
\hline \multirow{2}{*}{ ASIGNATURAS } & $2001 / 02$ & $2002 / 03$ & 2003/04 & $2004 / 05$ & $2005 / 06$ \\
\hline & $\%$ & $\%$ & $\%$ & $\%$ & $\%$ \\
\hline $\begin{array}{l}\text { Métodos, diseños y técnicas de } \\
\text { investigación psicológicos }\end{array}$ & 97 & 92 & 85 & 85 & 80 \\
\hline Procesos psicológicos básicos & 87 & 95 & 85 & 89 & 84 \\
\hline Psicología de la personalidad & 83 & 84 & 91 & 90 & 91 \\
\hline Psicología social & 91 & 86 & 93 & 94 & 88 \\
\hline $\begin{array}{l}\text { Diseño, desarrollo e innovación del } \\
\text { currículum }\end{array}$ & & 100 & 100 & 100 & 98 \\
\hline Diversidad en el grupo de iguales & & 100 & 100 & 97 & 93 \\
\hline Educación especial & & 88 & 100 & 100 & 100 \\
\hline Logopedia & & 96 & 93 & 86 & 94 \\
\hline $\begin{array}{l}\text { Malestar docente: ámbito de desarrollo e } \\
\text { intervención }\end{array}$ & & 100 & 100 & 97 & 95 \\
\hline Métodos de investigación en educación & & 96 & 75 & 92 & 83 \\
\hline $\begin{array}{l}\text { Modelos de orientación e intervención } \\
\text { psicopedagógica }\end{array}$ & & 89 & 85 & 89 & 88 \\
\hline
\end{tabular}




\begin{tabular}{|c|c|c|c|c|c|}
\hline \multirow{2}{*}{ ASIGNATURAS } & $2001 / 02$ & $2002 / 03$ & $2003 / 04$ & $2004 / 05$ & $2005 / 06$ \\
\hline & $\%$ & $\%$ & $\%$ & $\%$ & $\%$ \\
\hline Promoción y educación para la salud & & 100 & 100 & 100 & 96 \\
\hline Psicología de la instrucción & & 100 & 100 & 96 & 94 \\
\hline Psicología evolutiva & & 97 & 92 & 83 & 98 \\
\hline Diagnóstico en educación & & & 100 & 96 & 92 \\
\hline Diagnóstico psicológico & & & 92 & 100 & 96 \\
\hline $\begin{array}{l}\text { Dificultades de aprendizaje e intervención } \\
\text { psicopedagógica }\end{array}$ & & & 96 & 83 & 88 \\
\hline Fundamentos de evaluación & & & 93 & 95 & 95 \\
\hline Interacción y dinámica de grupos & & & 97 & 100 & 100 \\
\hline $\begin{array}{l}\text { Intervención psicopedagógica en los } \\
\text { trastornos del desarrollo }\end{array}$ & & & 98 & 96 & 94 \\
\hline Orientación profesional & & & 100 & 100 & 94 \\
\hline $\begin{array}{l}\text { Proyectos de intervención en pedagogía } \\
\text { social }\end{array}$ & & & 98 & 100 & 87 \\
\hline Practicum & & & 100 & 97 & 100 \\
\hline
\end{tabular}

Tabla 3.1. Resultados en psicopedagogía

\begin{tabular}{|l|l|}
\hline \multirow{2}{*}{ ASIGNATURAS } & $2005 / 06$ \\
\cline { 2 - 2 } & $\%$ \\
\hline Alemán I & 91 \\
\hline Alemán II & 100 \\
\hline Derecho de las empresas turísticas & 96 \\
\hline Estructura de mercados & 73 \\
\hline Inglés I & 86 \\
\hline Inglés II & 79 \\
\hline Introducción a la economía & 73 \\
\hline Ordenación administrativa del turismo & 89 \\
\hline Organización y gestión de empresas I & 75 \\
\hline Organización y gestión de empresas II & 85 \\
\hline Recursos territoriales turísticos I & 83 \\
\hline Recursos territoriales turísticos II & 82 \\
\hline
\end{tabular}

Tabla 3.2. Resultados en turismo 


\begin{tabular}{|l|l|}
\hline \multirow{2}{*}{ ASIGNATURAS } & $2005 / 06$ \\
\cline { 2 - 2 } & $\%$ \\
\hline Educación física y su didáctica & 97 \\
\hline Expresión plástica y su didáctica & 78 \\
\hline Idioma extranjero y su didáctica: Inglés & 96 \\
\hline Lengua española & 88 \\
\hline Lengua y literatura y su didáctica I & 99 \\
\hline Matemáticas y su didáctica & 88 \\
\hline Organización del centro escolar & 90 \\
\hline Psicología de la educación & 93 \\
\hline Psicología del desarrollo en edad escolar & 84 \\
\hline Sociología de la educación & 89 \\
\hline Teorías e instituciones contemporáneas de educación & 78 \\
\hline
\end{tabular}

Tabla 3.3. Resultados en magisterio

\section{Valoración de los estudiantes}

A finales de cada curso académico ponemos a disposición de los estudiantes una sencilla encuesta de valoración que contestan vía Web de manera voluntaria. A continuación exponemos un resumen de los principales resultados que arrojó esta encuesta el curso pasado (Castro, 2006).

Las edades de los estudiantes oscilan entre los 18 a los 59 años y se matriculan un mayor número de jóvenes de menor edad en la titulación de Turismo, elevándose un poco la edad de los encuestados en la de Maestro de Primaria y aumentando considerablemente en la licenciatura de Psicopedagogía, puesto que, el 25\% de los alumnos matriculados son mayores de 44 años (Ver Tabla 4).

\begin{tabular}{|l|l|l|l|}
\hline $\bar{X}$ & Turismo & Magisterio & Psicopedagogía \\
\hline Edad & 28,13 años & 30,76 años & 3,42 años \\
\hline
\end{tabular}

Tabla 4. Media de edad según titulaciones

En cuanto al género, en Turismo existe similar proporción entre mujeres y hombres, en cambio en Maestro el 77,8\% son mujeres y en Psicopedagogía un 74\%, lo que nos muestra una diferencia considerable (Ver Tabla 5). 


\begin{tabular}{|l|l|l|l|}
\hline & Turismo & Magisterio & Psicopedagogía \\
\hline Hombre & $46,7 \%$ & $22,2 \%$ & $26 \%$ \\
\hline Mujer & $53,3 \%$ & $77,8 \%$ & $74 \%$ \\
\hline
\end{tabular}

Tabla 5. Distribución de las titulaciones según género

Con respecto a la situación laboral, en Turismo y Magisterio más del 20\% de estudiantes sólo se dedican a sus estudios frente al apenas $2 \%$ de Psicopedagogía y son trabajadores por cuenta ajena más de la mitad del total de los estudiantes, aunque mayoritariamente lo hacen un 86\% de los estudiantes de Psicopedagogía (Ver Tabla 6).

\begin{tabular}{|l|l|l|l|}
\hline & Turismo & Magisterio & Psicopedagogía \\
\hline Trabaja por cuenta ajena & $57,1 \%$ & $65,1 \%$ & $86 \%$ \\
\hline Trabaja por cuenta propia & $7,1 \%$ & $7,0 \%$ & $2 \%$ \\
\hline Parado & $7,1 \%$ & $4,7 \%$ & $4 \%$ \\
\hline Pensionista & - & $2,3 \%$ & $2 \%$ \\
\hline Trabajo no remunerado & - & - & $4 \%$ \\
\hline Sólo estudia & $28,6 \%$ & $20,9 \%$ & $2 \%$ \\
\hline
\end{tabular}

Tabla 6: Distribución de las titulaciones según situación laboral

A la hora de indicar los motivos por los que eligieron esta forma de aprender, en Psicopedagogía un 75\% argumenta la ampliación de conocimientos además de una posible mejora en el trabajo en un $44,2 \%$. La mejora laboral en un $50 \%$, la ampliación de conocimientos en el 45,7\% y el aspecto vocacional en el 39,1\% de los casos son razones de peso en la titulación de Maestro. En Turismo se distribuyen proporcionalmente siendo significativo un $26,7 \%$ que afirman estudiar esta titulación para obtener un título universitario (Ver Tabla 7).

\begin{tabular}{|l|l|l|l|}
\hline Ítems & Turismo & Magisterio & Psicopedagogía \\
\hline Para ampliar los conocimientos & $26,7 \%$ & $45,7 \%$ & $75,0 \%$ \\
\hline Para mejorar en el trabajo & $33,3 \%$ & $50,0 \%$ & $44,2 \%$ \\
\hline Por vocación & $6,7 \%$ & $39,1 \%$ & $30,8 \%$ \\
\hline Porque disponen de tiempo y motivación por aprender & $26,7 \%$ & $23,9 \%$ & $25,0 \%$ \\
\hline Finalizar la titulación que comenzó en la presencial & $13,3 \%$ & $19,6 \%$ & $3,8 \%$ \\
\hline Para obtener un título universitario & $26,7 \%$ & $10,9 \%$ & $7,7 \%$ \\
\hline Para ganar más dinero & $13,3 \%$ & $10,9 \%$ & $1,9 \%$ \\
\hline
\end{tabular}

Tabla 7. Distribución de motivos por los que estudia una titulación universitaria 
Los tres colectivos de estudiantes son coincidentes a la hora de explicar porqué eligieron teleformación para estudiar, la falta de tiempo para asistir a clase y la imposibilidad de compatibilizar el trabajo y asistir a clase (Ver Tabla 8).

\begin{tabular}{|l|l|}
\hline Ítems & Porcentaje \\
\hline Imposibilidad de compatibilizar trabajo y asistir a clase & 54,0 \\
\hline Falta de tiempo para asistir a clase & 52,2 \\
\hline Falta de nota para acceder en modalidad presencial & 16,8 \\
\hline Por lejanía del Centro universitario & 14,2 \\
\hline Preferencias a estudiar a distancia & 9,7 \\
\hline Otros motivos & 8,0 \\
\hline
\end{tabular}

Tabla 8. Razones por las que estudian una titulación en modalidad no presencial

Mayoritariamente se conectan y acceden al Campus Virtual, el 85,8\%, desde la propia casa y el tipo de conexión que utilizan es ADSL (Ver Tabla 9).

\begin{tabular}{|l|l|}
\hline Ítems & Porcentaje \\
\hline Desde la propia casa & 85,8 \\
\hline Desde el trabajo & 8,8 \\
\hline Desde lugares públicos (bibliotecas, universidad, etc.) & 6,2 \\
\hline Desde la casa de familiar y/o amigos & 3,5 \\
\hline Desde un cibercafé & 1,8 \\
\hline
\end{tabular}

Tabla 9. Lugar habitual de acceso a Internet

En cuanto a la frecuencia de acceso al Campus Virtual, un 36,5\% de los estudiantes lo hacen casi todos los días, seguido del 27,9\% varías veces durante el día y el $26 \%$ que acceden varias veces a la semana (Ver Tabla 10).

\begin{tabular}{|l|l|}
\hline Ítems & Porcentaje \\
\hline Varias veces al mes & 4,8 \\
\hline Una vez a la semana & 4,8 \\
\hline Varias veces a la semana & 26,0 \\
\hline Casi todos los días & 36,5 \\
\hline Varias veces al día & 27,9 \\
\hline
\end{tabular}

Tabla 10: Frecuencia de acceso al Campus Virtual 
Habitualmente, los estudiantes acceden al Campus Virtual ULPGC de lunes a viernes en horario de tarde-noche y los de semana en horario de tarde y algo menos en la noche (Ver Tabla 11).

\begin{tabular}{|l|l|l|l|}
\hline Ítems & Mañana & Tarde & Noche \\
\hline De lunes a viernes & $22,1 \%$ & $56,6 \%$ & $46,0 \%$ \\
\hline Fin de semana & $28,3 \%$ & $40,7 \%$ & $31,9 \%$ \\
\hline
\end{tabular}

Tabla 11. Distribución de horario de conexión habitual al Campus Virtual

Un 32\% dedica semanalmente a los estudios entre 5-10 horas, el 28,4\% entre 1020 horas y menos de 5 horas semanales el 25,7\%. (Ver Tabla 12).

\begin{tabular}{|l|l|}
\hline Ítems & Porcentajes \\
\hline Menos de 5 horas & 25,7 \\
\hline Entre 5 y 10 horas & 31,9 \\
\hline Entre 10 y 15 horas & 14,2 \\
\hline Entre 15 y 20 horas & 14,2 \\
\hline Entre 20 y 25 horas & 7,1 \\
\hline Entre 25 y 30 horas & 4,4 \\
\hline Entre 35 y 40 horas & 0,9 \\
\hline Más de 40 horas & 1,8 \\
\hline
\end{tabular}

Tabla 12. Distribución de horas de dedicación semanal

Con respecto a las ventajas de estudiar a través de teleformación, el 90,3\% afirman sentirse partícipe de una comunidad virtual, el 74,3\% señalan la posibilidad de compatibilizar la formación con el trabajo y más de la mitad de los estudiantes indican la ventaja de estudiar sin necesidad de desplazarse $(55,8 \%)$ y aprender a su propio ritmo de trabajo $(52,2 \%)$ (Ver Tabla 13$)$. 


\begin{tabular}{|l|l|}
\hline Ítems & Porcentajes \\
\hline Sentirse participe de una comunidad virtual & 90,3 \\
\hline Posibilidad de compatibilizar la formación con el trabajo & 74,3 \\
\hline Estudiar sin necesidad de desplazarse & 55,8 \\
\hline Aprender y estudiar a su propio ritmo personal & 52,2 \\
\hline Posibilidad de compatibilizar la formación con la dedicación familiar & 28,3 \\
\hline Estudiar de forma autónoma necesitando menos la ayuda del profesor/a & 23,9 \\
\hline La alta calidad de la enseñanza a través de Internet & 8,0 \\
\hline
\end{tabular}

Tabla 13. Ventajas de estudiar y formarse a través de Internet

Para finalizar, los estudiantes han determinado en una escala likert de intervalo de 1 a 5 el grado de acuerdo que representa la eficacia de algunos recursos disponibles en el Campus Virtual. Según ellos las tutorías virtuales (diálogos) con el profesor es el recurso más eficaz para el estudio de las asignaturas alcanzando una media de 4,25 y seguido de las preguntas de autoevaluación, las consultas que exponen en los foros y las tareas (recurso que disponen para el envío y la calificación de las actividades) (Ver Tabla 14).

\begin{tabular}{|l|l|l|l|l|l|l|}
\hline \multirow{2}{*}{ RECURSOS } & \multicolumn{7}{|l|}{ Grado de acuerdo } & \multirow{2}{*}{} \\
\cline { 2 - 8 } & $\mathbf{1}$ & $\mathbf{2}$ & $\mathbf{3}$ & $\mathbf{4}$ & $\mathbf{5}$ & $\mathrm{X}$ \\
& $\mathbf{\%}$ & $\mathbf{\%}$ & $\mathbf{\%}$ & $\mathbf{\%}$ & $\mathbf{\%}$ & \\
\hline Consultas en el foro & - & 6,1 & 23,2 & 43,4 & 27,3 & 3,79 \\
\hline Diálogos con el tutor/a & 1,0 & 1,0 & 14,4 & 44,3 & 39,2 & 4,25 \\
\hline Sesiones presenciales & 8,5 & 11,0 & 35,4 & 34,1 & 11,0 & 3,22 \\
\hline Consultas telefónicas & 30,0 & 15,0 & 31,7 & 18,3 & 5,0 & 2,63 \\
\hline Tareas & 3,1 & 8,3 & 28,1 & 51,0 & 9,4 & 3,50 \\
\hline Preguntas de autoevaluación & 1,1 & 7,5 & 19,4 & 41,9 & 30,1 & 3,91 \\
\hline
\end{tabular}

Tabla 14. Grado de eficacia de los recursos disponibles para el desarrollo de las asignaturas

\section{ENSEÑANZA PRESENCIAL}

La apuesta de la ULPGC por la implantación de estudios en modalidad no presencial a través de teleformación pronto "contagió" sus virtudes a la enseñanza presencial y muy pronto los profesores solicitaron poder utilizarla también como apoyo para sus asignaturas presenciales. 
A lo largo del curso 2003/2004 y dentro del Plan de Formación del Profesorado de la ULPGC, los Vicerrectorados de Planificación y Calidad; y Desarrollo Institucional y Nuevas Tecnologías colaboraron para desarrollar conjuntamente un curso sobre "Entornos Virtuales de Aprendizaje (EVA) como apoyo a la enseñanza presencial".

Debido a la enorme demanda recibida por parte del profesorado, se llevaron a cabo 9 ediciones y un total de 360 profesores se inscribieron en los cursos y, tras una sesión presencial donde se iniciaban en el manejo del Campus Virtual de la ULPGC, continuaron el curso a través de Internet. El curso tenía como objetivos que los docentes identificaran las características básicas de un Entorno Virtual de Aprendizaje (EVA) y de las Plataformas de Teleformación, además de conocer y dominar las herramientas del Campus Virtual de la ULPGC.

De este modo, los profesores tuvieron la oportunidad de conocer conceptualmente y de manera práctica, las modalidades de formación donde se combina la presencialidad con la distancia, las principales herramientas que debe incluir un EVA (contenidos, foros, buzón personal, etc.) y los soportes tecnológicos donde se desarrollan los procesos formativos, especialmente, el Campus Virtual de la ULPGC desde donde se impartía dicho curso. Por tanto, los docentes que realizaban el curso vivieron la experiencia de explorar las características generales que ofrecía esta herramienta educativa, los requisitos mínimos exigidos (hardware y software, el proveedor de Internet, los tipos de conexión y navegadores) su acceso y funcionamiento y, por último, poner en práctica las recomendaciones para una comunicación efectiva en TIC.

Al final del curso 2003/2004, 280 profesores habían culminado con éxito esta formación:

\begin{tabular}{|l|l|l|l|l|l|}
\hline & Área1 & Área2 & Área3 & Área4 & Total \\
\hline Número total de profesores de la ULPGC por áreas & 385 & 425 & 532 & 203 & 1545 \\
\hline Porcentaje con respecto al total de la ULPGC & 24,9 & 27,5 & 34,4 & 13,1 & 100 \\
\hline Número de profesores que terminaron el curso & 44 & 99 & 98 & 39 & 280 \\
\hline Porcentaje con respecto al total del área & 11,4 & 23,3 & 18,4 & 19,2 & 18,1 \\
\hline Porcentaje con respecto a los que terminaron el curso & 15,7 & 35,4 & 35,0 & 13,9 & 100 \\
\hline
\end{tabular}

Tabla 15. Distribución según profesorado por áreas

En el segundo cuatrimestre del curso 2003/04 se puso en marcha un Plan experimental de apoyo a la enseñanza presencial tras el éxito de los cursos de 
formación y la demanda del profesorado. En esta primera convocatoria, se inscribieron 85 profesores para impartir 76 asignaturas.

\begin{tabular}{|l|c|}
\hline FACULTAD/ESCUELA & $\mathbf{N}^{\mathbf{0}}$ \\
\hline Escuela Técnica Superior de Arquitectura & 5 \\
\hline Escuela Técnica Superior de Ingeniería Industrial & 1 \\
\hline Escuela Técnica Superior de Ingenieros de Telecomunicación & 7 \\
\hline Escuela Universitaria de Informática & 4 \\
\hline Escuela Universitaria de Ingeniería Técnica de Telecomunicación & 6 \\
\hline Escuela Universitaria Politécnica & 8 \\
\hline Facultad de Ciencias de la Actividad Física y el Deporte & 2 \\
\hline Facultad de Ciencias de la Salud & 5 \\
\hline Facultad de Ciencias del Mar & 2 \\
\hline Facultad de Ciencias Económicas y Empresariales & 5 \\
\hline Facultad de Ciencias Jurídicas & 3 \\
\hline Facultad de Filología & 4 \\
\hline Facultad de Formación del Profesorado & 8 \\
\hline Facultad de Geografía e Historia & 3 \\
\hline Facultad de Informática & 5 \\
\hline Facultad de Traducción e Interpretación & 4 \\
\hline Facultad de Veterinaria & 4 \\
\hline Total ULPGC & 76 \\
\hline
\end{tabular}

Tabla 16. Distribución fr asignaturas con apoyo TIC por facultades/escuelas en el curso 2003/2004

Al final del curso 2003/2004 de los 1545 profesores/as que conformaban la plantilla de la ULPGC, unos $327(21,17)$ habían participado en alguna de las siguientes acciones relacionadas con el uso de las TIC para la docencia:

Curso de formación sobre "Entornos Virtuales de Aprendizaje (EVA) como apoyo a la enseñanza presencial”.

Como docentes en algunas de las acciones de teleformación (psicopedagogía en línea, doctorado, posgrados, extensión universitaria, formación continua, etc.).

Como docentes que han apoyado alguna de sus asignaturas presenciales con la Plataforma Educativa de la ULPGC. 
Así, la distribución por áreas de los docentes respecto al total de la Universidad de Las Palmas de Gran Canaria se expresa en la siguiente tabla:

\begin{tabular}{|l|l|l|l|l|l|}
\hline & Área 1 & Área 2 & Área 3 & Área 4 & Total \\
\hline Número total de profesores de la ULPGC por áreas & 385 & 425 & 532 & 203 & 1545 \\
\hline Porcentaje con respecto al total de la ULPGC & 24,9 & 27,5 & 34,4 & 13,1 & 100 \\
\hline Numero de profesores con algún tipo experiencia & 45 & 134 & 108 & 40 & 327 \\
\hline Porcentaje con respecto al total del área & 11,7 & 31,5 & 20,3 & 19,7 & 21,2 \\
\hline Porcentaje con respecto al total con experiencia & 13,8 & 41,0 & 33,0 & 12,2 & 100 \\
\hline
\end{tabular}

Tabla 17. Distribución según experiencia en TIC del profesorado por áreas en el curso 2003/2004

Desde el curso 2004-2005 todas las asignaturas de las diferentes carreras en modalidad presencial que se imparten en ULPGC tienen a su disposición una plataforma de teleformación de apoyo que incorpora todos los servicios propios de este tipo de aplicaciones. Así, además de repositorios de documentos de texto y multimedia, se ofrecen otras herramientas más avanzadas como foros, tablones de anuncios, tutorías, correo electrónico, chats, etc.

A través de este entorno, los estudiantes pueden contactar con sus profesores, plantear sus dudas, enviar sus trabajos y recibir las correspondientes calificaciones, etc.

Como se puede ver en la siguiente tabla, un curso más tarde, se incrementó en 400 el número de asignaturas que utilizaban el Campus Virtual.

\begin{tabular}{|c|c|}
\hline FACULTAD/ESCUELA & $\mathbf{N}^{\circ}$ \\
\hline Escuela Técnica Superior de Arquitectura & 15 \\
\hline Escuela Técnica Superior de Ingeniería Industrial & 31 \\
\hline Escuela Técnica Superior de Ingenieros de Telecomunicación & 20 \\
\hline Escuela Universitaria de Informática & 15 \\
\hline Escuela Universitaria de Ingeniería Técnica de Telecomunicación & 31 \\
\hline Escuela Universitaria Politécnica & 70 \\
\hline Facultad de Ciencias de la Actividad Física y el Deporte & 17 \\
\hline Facultad de Ciencias de la Salud & 31 \\
\hline Facultad de Ciencias del Mar & 21 \\
\hline
\end{tabular}




\begin{tabular}{|l|l|}
\hline FACULTAD/ESCUELA & $\mathbf{N}^{\mathbf{0}}$ \\
\hline Facultad de Ciencias Económicas y Empresariales & 50 \\
\hline Facultad de Ciencias Jurídicas & 30 \\
\hline Facultad de Filología & 15 \\
\hline Facultad de Formación del Profesorado & 67 \\
\hline Facultad de Geografía e Historia & 14 \\
\hline Facultad de Informática & 17 \\
\hline Facultad de Traducción e Interpretación & 10 \\
\hline Facultad de Veterinaria & 22 \\
\hline Total ULPGC & $\mathbf{4 7 6}$ \\
\hline
\end{tabular}

Tabla 18. Distribución de asignaturas apoyadas en TIC por facultades y escuelas en el curso 2004/2005

Como ejemplo del uso de este tipo de apoyo para la docencia y el aprendizaje, la siguiente tabla recoge el número de profesores y estudiantes que utilizaron de manera activa las TIC como apoyo a la enseñanza presencial durante el curso 2004/2005, aproximadamente, la cuarta parte del profesorado y un tercio de los estudiantes.

\begin{tabular}{|l|l|l|}
\hline FACULTADES/ESCUELAS & PROFESORES & ESTUDIANTES \\
\hline Escuela Técnica Superior de Arquitectura & 19 & 521 \\
\hline Escuela Técnica Superior de Ingeniería Industrial & 25 & 324 \\
\hline Escuela Técnica Superior de Ingenieros de Telecomunicación & 19 & 205 \\
\hline Escuela Universitaria de Informática & 15 & 243 \\
\hline Escuela Universitaria de Ingeniería Técnica de Telecomunicación & 27 & 346 \\
\hline Escuela Universitaria Politécnica & 47 & 873 \\
\hline Facultad de Ciencias de la Actividad Física y el Deporte & 8 & 151 \\
\hline Facultad de Ciencias de la Salud & 31 & 583 \\
\hline Facultad de Ciencias del Mar & 18 & 297 \\
\hline Facultad de Ciencias Económicas y Empresariales & 56 & 1.734 \\
\hline Facultad de Ciencias Jurídicas & 24 & 733 \\
\hline Facultad de Filología & 15 & 215 \\
\hline Facultad de Formación del Profesorado & 37 & 890 \\
\hline Facultad de Geografía e Historia & 9 & 162 \\
\hline Facultad de Informática & 12 & 141 \\
\hline Facultad de Traductores e Interpretes & 6 & 75 \\
\hline Facultad de Veterinaria & 31 & 398 \\
\hline Total ULPGC & 399 & $\mathbf{7 . 8 9 1}$ \\
\hline \hline RESPECTO AL TOTAL & $\mathbf{2 5 , 8 3 \%}$ & $\mathbf{3 4 , 5 2 \%}$ \\
\hline
\end{tabular}

Tabla 19. Número de profesores y estudiantes activos en el uso de las TIC en el curso 2004/2005 
Por último, en el curso 2005-2006, el incremento de asignaturas es de nuevo notable superando las 850 .

\begin{tabular}{|l|l|}
\hline FACULTAD/ESCUELA & $\mathbf{N}^{\circ}$ \\
\hline Escuela Técnica Superior de Arquitectura & 20 \\
\hline Escuela Técnica Superior de Ingeniería Industrial & 58 \\
\hline Escuela Técnica Superior de Ingenieros de Telecomunicación & 39 \\
\hline Escuela Universitaria de Informática & 19 \\
\hline Escuela Universitaria de Ingeniería Técnica de Telecomunicación & 80 \\
\hline Escuela Universitaria Politécnica & 133 \\
\hline Facultad de Ciencias de la Actividad Física y el Deporte & 23 \\
\hline Facultad de Ciencias de la Salud & 65 \\
\hline Facultad de Ciencias del Mar & 34 \\
\hline Facultad de Ciencias Económicas y Empresariales & 90 \\
\hline Facultad de Ciencias Jurídicas & 43 \\
\hline Facultad de Filología & 27 \\
\hline Facultad de Formación del Profesorado & 89 \\
\hline Facultad de Geografía e Historia & 59 \\
\hline Facultad de Informática & 26 \\
\hline Facultad de Traducción e Interpretación & 20 \\
\hline Facultad de Veterinaria & 30 \\
\hline Total ULPGC & 855 \\
\hline
\end{tabular}

Tabla 20. Distribución de asignaturas apoyadas en TIC por facultades y escuelas en el curso 2005/2006

De la misma manera, el número de usuarios activos también se ha incrementado, duplicando las cifras del curso anterior, alcanzando, en el caso del profesorado el 50 y superando el 60 en el caso de los estudiantes.

\begin{tabular}{|l|l|l|}
\hline FACULTADES/ESCUELAS & PROFESORES & ESTUDIANTES \\
\hline Escuela Técnica Superior de Arquitectura & 27 & 767 \\
\hline Escuela Técnica Superior de Ingeniería Industrial & 74 & 612 \\
\hline Escuela Técnica Superior de Ingenieros de Telecomunicación & 52 & 289 \\
\hline Escuela Universitaria de Informática & 39 & 636 \\
\hline Escuela Universitaria de Ingeniería Técnica de Telecomunicación & 66 & 624 \\
\hline Escuela Universitaria Politécnica & 116 & 1677 \\
\hline F. de Ciencias de la Actividad Física y el Deporte & 23 & 379 \\
\hline Facultad de Ciencias de la Salud & 69 & 894 \\
\hline
\end{tabular}




\begin{tabular}{|l|l|l|}
\hline FACULTADES/ESCUELAS & PROFESORES & ESTUDIANTES \\
\hline Facultad de Ciencias del Mar & 41 & 365 \\
\hline Facultad de Ciencias Económicas y Empresariales & 113 & 2871 \\
\hline Facultad de Ciencias Jurídicas & 55 & 1651 \\
\hline Facultad de Filología & 37 & 368 \\
\hline Facultad de Formación del Profesorado & 58 & 1273 \\
\hline Facultad de Geografía e Historia & 38 & 400 \\
\hline Facultad de Informática & 32 & 420 \\
\hline Facultad de Traductores e Interpretes & 30 & 410 \\
\hline Facultad de Veterinaria & 44 & 474 \\
\hline Total ULPGC & 914 & 14.110 \\
\hline \hline RESPECTO AL TOTAL & $49,97 \%$ & $61,72 \%$ \\
\hline
\end{tabular}

Tabla 21. Número de profesores y estudiantes activos en el uso de las TIC en el curso 2005/2006

La siguiente tabla resume la evolución desde el curso 2003-2004 hasta el curso 2005-2006 en cuanto al número de asignaturas con apoyo TIC.

\begin{tabular}{|l|l|l|l|}
\hline FACULTADES/ESCUELAS & $\mathbf{0 3 / 0 4}$ & $\mathbf{0 4 / 0 5}$ & $\mathbf{0 5 / 0 6}$ \\
\hline Escuela Técnica Superior de Arquitectura & 5 & 15 & 20 \\
\hline Escuela Técnica Superior de Ingeniería Industrial & 1 & 31 & 58 \\
\hline Escuela Técnica Superior de Ingenieros de Telecomunicación & 7 & 20 & 39 \\
\hline Escuela Universitaria de Informática & 4 & 15 & 19 \\
\hline Escuela Universitaria de Ingeniería Técnica de Telecomunicación & 6 & 31 & 80 \\
\hline Escuela Universitaria Politécnica & 8 & 70 & 133 \\
\hline Facultad de Ciencias de la Actividad Física y el Deporte & 2 & 17 & 23 \\
\hline Facultad de Ciencias de la Salud & 5 & 31 & 65 \\
\hline Facultad de Ciencias del Mar & 2 & 21 & 34 \\
\hline Facultad de Ciencias Económicas y Empresariales & 5 & 50 & 90 \\
\hline Facultad de Ciencias Jurídicas & 3 & 30 & 43 \\
\hline Facultad de Filología & 4 & 15 & 27 \\
\hline Facultad de Formación del Profesorado & 8 & 67 & 89 \\
\hline Facultad de Geografía e Historia & 3 & 14 & 59 \\
\hline Facultad de Informática & 5 & 17 & 26 \\
\hline Facultad de Traducción e Interpretación & 4 & 10 & 20 \\
\hline Facultad de Veterinaria & 4 & 22 & 30 \\
\hline Total ULPGC & 76 & 476 & 855 \\
\hline
\end{tabular}

Tabla 22. Evolución del número de asignaturas con apoyo TIC, cursos 2003/2004, 2004/2005 y 2005/2006 
De la misma manera, las siguientes tablas resumen la evolución en cuanto al número de profesores y estudiantes activos en el uso de las TIC en los dos últimos cursos. En la de profesores se puede observar que se pasó de un curso a otro de un 25 a un 50 .

\begin{tabular}{|l|l|l|}
\hline FACULTADES/ESCUELAS & $04 / 05$ & $05 / 06$ \\
\hline Escuela Técnica Superior de Arquitectura & 19 & 27 \\
\hline Escuela Técnica Superior de Ingeniería Industrial & 25 & 74 \\
\hline Escuela Técnica Superior de Ingenieros de Telecomunicación & 19 & 52 \\
\hline Escuela Universitaria de Informática & 15 & 39 \\
\hline Escuela Universitaria de Ingeniería Técnica de Telecomunicación & 27 & 66 \\
\hline Escuela Universitaria Politécnica & 47 & 116 \\
\hline Facultad de Ciencias de la Actividad Física y el Deporte & 8 & 23 \\
\hline Facultad de Ciencias de la Salud & 31 & 69 \\
\hline Facultad de Ciencias del Mar & 18 & 41 \\
\hline Facultad de Ciencias Económicas y Empresariales & 56 & 113 \\
\hline Facultad de Ciencias Jurídicas & 24 & 55 \\
\hline Facultad de Filología & 15 & 37 \\
\hline Facultad de Formación del Profesorado & 37 & 58 \\
\hline Facultad de Geografía e Historia & 9 & 38 \\
\hline Facultad de Informática & 12 & 32 \\
\hline Facultad de Traducción e Interpretación & 6 & 30 \\
\hline Facultad de Veterinaria & 31 & 44 \\
\hline Total ULPGC & 399 & 914 \\
\hline \hline RESPECTO AL TOTAL & $25,83 \%$ & $49,97 \%$ \\
\hline
\end{tabular}

Tabla 23. Evolución del número de profesores activos en el uso de las tic, cursos 2004/2005 y 2005/2006

En la de estudiantes podemos observar que se pasó de un curso a otro, de un tercio a, prácticamente, dos tercios.

\begin{tabular}{|l|l|l|}
\hline FACULTADES/ESCUELAS & $04 / 05$ & $05 / 06$ \\
\hline Escuela Técnica Superior de Arquitectura & 521 & 767 \\
\hline Escuela Técnica Superior de Ingeniería Industrial & 324 & 612 \\
\hline Escuela Técnica Superior de Ingenieros de Telecomunicación & 205 & 289 \\
\hline Escuela Universitaria de Informática & 243 & 636 \\
\hline Escuela Universitaria de Ingeniería Técnica de Telecomunicación & 346 & 624 \\
\hline
\end{tabular}




\begin{tabular}{|l|l|l|}
\hline FACULTADES/ESCUELAS & $04 / 05$ & $05 / 06$ \\
\hline Escuela Universitaria Politécnica & 873 & 1677 \\
\hline Facultad de Ciencias de la Actividad Física y el Deporte & 151 & 379 \\
\hline Facultad de Ciencias de la Salud & 583 & 894 \\
\hline Facultad de Ciencias del Mar & 297 & 365 \\
\hline Facultad de Ciencias Económicas y Empresariales & 1.734 & 2871 \\
\hline Facultad de Ciencias Jurídicas & 733 & 1651 \\
\hline Facultad de Filología & 215 & 368 \\
\hline Facultad de Formación del Profesorado & 890 & 1273 \\
\hline Facultad de Geografía e Historia & 162 & 400 \\
\hline Facultad de Informática & 141 & 420 \\
\hline Facultad de Traducción e Interpretación & 75 & 410 \\
\hline Facultad de Veterinaria & 398 & 474 \\
\hline Total ULPGC & 7.891 & 14.110 \\
\hline RESPECTO AL TOTAL & $34,52 \%$ & $61,72 \%$ \\
\hline
\end{tabular}

Tabla 24. Evolución del número de estudiantes activos en el uso de las TIC, cursos 2004/2005 y 2005/2006

$\mathrm{Al}$ mismo tiempo que se producido este notable incremento de usuarios curso tras curso, hemos evaluado las principales características del contexto en el que se han producido y las expectativas de los estudiantes hacia el uso de las TIC como apoyo a su enseñanza presencial.

Así por ejemplo, con respecto al lugar habitual de conexión la mayoría lo hace desde las salas de libre disposición de la universidad o desde su propia casa (Ver Tabla 25).

\begin{tabular}{|l|l|}
\hline Ítems & Porcentaje \\
\hline Salas de libre disposición de la universidad & 54,0 \\
\hline Ciber & 6,8 \\
\hline Propia casa & 44,7 \\
\hline Desde la casa de amigos, pareja, etc. & 10,8 \\
\hline Salas de libre disposición externa & 0,2 \\
\hline
\end{tabular}

Tabla 25. Desde qué lugar se conecta al Campus Virtual 
Más del 90\% puede acceder desde su propia casa, reciben apoyo en unas 4 asignaturas, lo que supone unos 24 créditos, a las que dedican casi 4 horas a la semana, de media, a trabajar a través del Campus Virtual de la ULPGC (descarga de contenidos, envío de mensajes, entrega de actividades, etc.).

Acceden habitualmente al Campus Virtual una o varias veces a la semana (65,6\%), aunque los hay que acceden todos los días incluso más de una vez al día (Ver Tabla 26).

\begin{tabular}{|l|l|}
\hline İtems & Porcentaje \\
\hline Menos de una vez a la semana & 16,1 \\
\hline Una vez a la semana & 33,4 \\
\hline Varias veces a la semana & 32,2 \\
\hline Casi todos los días & 12,8 \\
\hline Varias veces al día & 5,5 \\
\hline
\end{tabular}

Tabla 26. Frecuencia con la que accede al Campus Virtual

Se suelen conectar preferentemente de lunes a viernes en horario de mañanatarde y mucho menos los fines de semana (Ver Tabla 27).

\begin{tabular}{|l|l|l|l|l|l|}
\hline \multicolumn{4}{|l|}{ Entre semana } & \multicolumn{3}{l|}{ Fin de semana } \\
\hline mañana & tarde & noche & mañana & tarde & noche \\
\hline 56 & 54,1 & 29,4 & 23,2 & 22,7 & 15 \\
\hline
\end{tabular}

Tabla 27. Días de la semana y horario en que se conectan habitualmente al Campus Virtual

Cada vez que se conectan, la mayoría, dedican menos de 30 minutos (Ver Tabla 28).

\begin{tabular}{|l|l|}
\hline İtems & Porcentaje \\
\hline Menos de 30 minutos & 66,9 \\
\hline Entre 30 y 60 minutos & 29,0 \\
\hline Entre 1 hora y 2 horas & 4,1 \\
\hline
\end{tabular}

Tabla 28. Tiempo que dedican cada vez que se conecta

En cuanto a las utilidades disponibles, los primeros lugares lo ocupan la descarga de apuntes y contenidos, lectura de comunicados del profesor, envío de tareas y actividades, y consultas al profesor (Ver Tabla 29). 


\begin{tabular}{|l|l|}
\hline Ítems & Porcentaje \\
\hline Descarga de apuntes y contenidos de interés & 92,0 \\
\hline Lectura de avisos y comunicados del profesor y/o compañeros & 74,9 \\
\hline Consulta de dudas (tutorías) & 43,1 \\
\hline Contactos con los compañeros (mensajería) & 27,4 \\
\hline Envío de tareas y/o actividades & 48,1 \\
\hline Participación en foros de debate & 24,0 \\
\hline Participación en chats & 7,0 \\
\hline Tutoría concertada con el profesor & 15,9 \\
\hline Realización de pruebas autoevaluativas & 18,6 \\
\hline Calificaciones & 0,5 \\
\hline
\end{tabular}

Tabla 29. Apoyos que recibe y/o tiene disponible a través del Campus Virtual

Por último, curso tras curso preguntamos a los estudiantes por las consecuencias del uso generalizado de herramientas TIC (Castro y Chirino, 2004). A continuación mostramos los resultados de su última aplicación a finales del curso 2005-2006. Aunque las respuestas van desde 1 (totalmente en desacuerdo) hasta 5 (totalmente de acuerdo) en la tabla sólo hemos puesto la media y los porcentajes que corresponden a los grados de acuerdo más altos (muy de acuerdo y totalmente de acuerdo).

Así, en la categoría de las consecuencias del uso generalizado de herramientas TIC relacionadas con el profesor (Ver Tabla 30.1), los niveles generalizados de acuerdo se alcanzan en todos los ítems, donde coinciden tanto los profesores como los estudiantes en que el profesorado tendrá que formarse en nuevas estrategias de enseñanza.

\begin{tabular}{|c|c|c|c|c|}
\hline & \multicolumn{2}{|c|}{ Profesores } & \multicolumn{2}{|c|}{ Estudiantes } \\
\hline & $\bar{x}$ & $\begin{array}{l}4+5 \\
\%\end{array}$ & $\bar{x}$ & $\begin{array}{l}4+5 \\
\%\end{array}$ \\
\hline El profesorado tendrá que formarse en nuevas estrategias de enseñanza & 4.18 & 81,2 & 3.80 & 61,9 \\
\hline El profesor tendrá que cambiar de rol (funciones) & 3.69 & 59,6 & 3.22 & 38,2 \\
\hline Generará más trabajo y esfuerzo para el profesor & 4.11 & 77,6 & 3.12 & 34,5 \\
\hline
\end{tabular}

Tabla 30.1. Consecuencias relacionadas con el profesor

Como consecuencias del uso de TIC en la enseñanza relacionadas con el estudiante (Ver Tabla 30.2), profesores y estudiantes coinciden en que los estudiantes tendrán que estar atentos a más fuentes de información. 


\begin{tabular}{|c|c|c|c|c|}
\hline & \multicolumn{2}{|c|}{ Profesores } & \multicolumn{2}{|c|}{ Estudiantes } \\
\hline & $\bar{x}$ & $\begin{array}{l}4+5 \\
\%\end{array}$ & $\bar{x}$ & $\begin{array}{l}4+5 \\
\%\end{array}$ \\
\hline $\begin{array}{l}\text { Generará desconcierto porque para estar informado habrá que estar } \\
\text { atento a otras fuentes de información además de la clase presencial }\end{array}$ & 2.29 & 13,2 & 2.91 & 29,2 \\
\hline $\begin{array}{l}\text { Dividirá el grupo-clase entre los que la utilicen con frecuencia y los que } \\
\text { no suelan acceder a ella }\end{array}$ & 2.75 & 28,4 & 2.93 & 40,5 \\
\hline Generará más trabajo y esfuerzo para los estudiantes & 3.05 & 34,8 & 3.04 & 32,1 \\
\hline Los estudiantes tendrán que estar atentos a más fuentes de información & 3.74 & 68,5 & 3.57 & 51,1 \\
\hline
\end{tabular}

Tabla 3o. 2. Consecuencias relacionadas con el estudiante

Respecto a la calidad de la enseñanza a la vista de los resultados de la Tabla 30.3, tanto profesores como estudiantes se manifiestan de acuerdo con que mejorará de manera sustancial la calidad de la enseñanza. Además, se muestran en desacuerdo (medias inferiores a 3) respecto a la afirmación tendrá más un uso de tipo social o lúdico que académico, aunque, no obstante existen diferencias en los ítems supondrá una pérdida de tiempo y no aportará nada nuevo y la calidad de la enseñanza será la misma, donde los profesores se muestran mayormente en desacuerdo con tales afirmaciones a diferencia de los estudiantes.

\begin{tabular}{|c|c|c|c|c|}
\hline & \multicolumn{2}{|c|}{ Profesores } & \multicolumn{2}{|c|}{ Estudiantes } \\
\hline & $\bar{x}$ & $\begin{array}{l}4+5 \\
\%\end{array}$ & $\bar{x}$ & $\begin{array}{l}4+5 \\
\%\end{array}$ \\
\hline No aportará nada nuevo, la calidad de la enseñanza será la misma & 1.85 & 6,3 & 2.55 & 19,4 \\
\hline Mejorará de manera sustancial la calidad de la enseñanza & 3.51 & 53,1 & 3.37 & 43,9 \\
\hline Supondrá una pérdida de tiempo & 1.59 & 3,5 & 2.35 & 17,2 \\
\hline Tendrá más un uso de tipo social o lúdico que académico & 1.92 & 4,8 & 2.62 & 20,7 \\
\hline
\end{tabular}

Tabla 30.3. Consecuencias relacionadas con la calidad de la enseñanza

En la valoración de las propias herramientas de las tecnologías de la información y comunicación (Ver Tabla 30.4), existen diferencias entre los estudiantes y el profesorado puesto que, mientras el $73 \%$ del profesorado se manifiesta en niveles altos y muy altos de acuerdo al ítem sera necesario un equipamiento informático adecuado, los estudiantes bajan al 46,9.

Sí parece existir mayor coicidencia entre los profesores y estudiantes con un nivel de acuerdo generalizado respecto a que se ampliará de manera adicional el conocimiento sobre las tecnologías de la información y la comunicación y en el 
caso de los estudiantes tendrán que hacer un esfuerzo (comprar ordenador, ir a un Ciber, salas de libre disposición, etc.) para acceder a Internet.

\begin{tabular}{|l|l|l|l|l|l|}
\hline & \multicolumn{2}{l|}{ Profesores } & \multicolumn{2}{l|}{ Estudiantes } \\
\cline { 2 - 6 } & $\begin{array}{l}\text { X } \\
\text { X }\end{array}$ & $\begin{array}{l}4+5 \\
\%\end{array}$ & $\bar{X}$ & $\begin{array}{l}4+5 \\
\%\end{array}$ \\
\hline $\begin{array}{l}\text { Ampliará de manera adicional el conocimiento sobre las tecnologías de la } \\
\text { información y la comunicación }\end{array}$ & 4.01 & 75,0 & 3.61 & 53,7 \\
\hline $\begin{array}{l}\text { Los estudiantes tendrán que hacer un esfuerzo (comprar ordenador, ir a } \\
\text { un Ciber, salas de libre disposición, etc.) para acceder a Internet }\end{array}$ & 3.51 & 56,1 & 3.45 & 48,8 \\
\hline Será necesario un equipamiento informático adecuado & 4.16 & 77,9 & 3.58 & 42,0 \\
\hline Será necesario unos conocimientos mínimos sobre el manejo de TIC & 3.96 & 73,0 & 3.46 & 46,9 \\
\hline
\end{tabular}

Tabla 30.4. Consecuencias relacionadas con las TIC

Observando las medias del nivel de acuerdo respecto a las consecuencias generalizadas de herramientas TIC relacionadas con los contenidos (Ver Tabla 30.5), se puede observar un nivel de acuerdo moderado en este apartado entre profesores y estudiantes. De esta manera, aunque ciertos contenidos seguirán siendo difíciles de explicar/entender de manera no presencial las TIC facilitarán un mayor y mejor acceso a los contenidos e incluso facilitarán la comprensión de los contenidos.

\begin{tabular}{|l|l|l|l|l|}
\hline & \multicolumn{2}{|l|}{ Profesores } & \multicolumn{2}{l|}{ Estudiantes } \\
\cline { 2 - 5 } & & $\begin{array}{l}4+5 \\
\%\end{array}$ & \multicolumn{4}{l}{$\begin{array}{l}4+5 \\
\%\end{array}$} \\
\hline Facilitará la comprensión de los contenidos & 3.52 & 55,0 & 3.41 & 45,3 \\
\hline $\begin{array}{l}\text { Ciertos contenidos seguirán siendo difíciles de explicar/entender de } \\
\text { manera no presencial }\end{array}$ & 4.07 & 73,8 & 3.67 & 55,5 \\
\hline Facilitará un mayor y mejor acceso a los contenidos & 4.08 & 76,8 & 3.73 & 58,5 \\
\hline
\end{tabular}

Tabla 30.5. Consecuencias relacionadas con los contenidos

En la Tabla 30.6 se puede establecer que, tanto los profesores como estudiantes se manifiestan en la categoría de las consecuencias del uso generalizado de herramientas TIC relacionadas con la comunicación e interacción, en niveles de acuerdo moderados superior al punto medio exceptuando el ítem disminuirán las relaciones sociales presenciales, puesto que si bien los profesores indican desacuerdo con una puntuación media inferior a 3, los estudiantes se muestran moderadamente de acuerdo con puntuación superior al punto medio. 
De esta manera, profesores y estudiantes muestran puntuaciones similares en el resto de los ítems que conforman esta categoría, por tanto, indicando un acuerdo moderado respecto a los ítems que la conforman.

\begin{tabular}{|c|c|c|c|c|}
\hline & \multicolumn{2}{|c|}{ Profesores } & \multicolumn{2}{|c|}{ Estudiantes } \\
\hline & $\bar{x}$ & $\begin{array}{l}4+5 \\
\%\end{array}$ & $\bar{x}$ & $\begin{array}{l}4+5 \\
\%\end{array}$ \\
\hline $\begin{array}{l}\text { Aumentará el número de interacciones de los estudiantes } \\
\text { entre si }\end{array}$ & 3.26 & 40,4 & 3.32 & 43,3 \\
\hline $\begin{array}{l}\text { Aumentará el número de interacciones entre el profesor y los } \\
\text { estudiantes }\end{array}$ & 3.71 & 61,3 & 3.41 & 47,1 \\
\hline Disminuirán las relaciones sociales presenciales & 2.75 & 25,7 & 3.08 & 35,2 \\
\hline Fomentará el trabajo colaborativo entre los estudiantes & 2.98 & 29,1 & 3.03 & 30,8 \\
\hline El proceso de enseñanza-aprendizaje será más personalizado & 3.37 & 47,3 & 3.19 & 38,0 \\
\hline Mejorará la comunicación con el profesor & 3.67 & 60,2 & 3.29 & 41,4 \\
\hline Será más fácil expresar opiniones & 3.59 & 55,7 & 3.42 & 46,4 \\
\hline Mejorará la comunicación entre los estudiantes & 3.18 & 36,1 & 3.13 & 33,8 \\
\hline Será más fácil plantear dudas/consultas & 3.87 & 71,6 & 3.59 & 54,0 \\
\hline
\end{tabular}

Tabla 3o.6. Consecuencias relacionadas con la comunicación e interacción

Por último, en la tabla 30.7 y como consecuencias derivadas de uso generalizado de las TIC relacionadas con la presencialidad, tanto profesores como estudiantes coinciden y reconocen al valorar en un alto nivel de acuerdo (superior a 4 en los profesores y cerca del mismo valor los estudiantes) que permitirá hacer consultas sin desplazamientos.

No obstante, mientras que para el profesorado los ítems: descenderá el número de estudiantes que irán a las tutorías y ya no será necesario asistir tanto a las tutorías; presentan medias inferiores a 3 y por tanto, se muestran en desacuerdo con tales afirmaciones, no es compartida su opinión entre los estudiantes puesto que para ellos las mismas afirmaciones se sitúan con una media superior al punto medio. Además, mientras que el profesorado valora el ítem no será necesaria la asistencia presencial con una puntuación media inferior a 2 mostrándose en desacuerdo, los estudiantes presentan una media superior a la misma. 


\begin{tabular}{|c|c|c|c|c|}
\hline & \multicolumn{2}{|c|}{ Profesores } & \multicolumn{2}{|c|}{ Estudiantes } \\
\hline & $\bar{x}$ & $\begin{array}{l}4+5 \\
\%\end{array}$ & $\bar{x}$ & $\begin{array}{l}4+5 \\
\%\end{array}$ \\
\hline $\begin{array}{l}\text { Descenderá el número de estudiantes que asistan a las clases } \\
\text { presenciales }\end{array}$ & 2.89 & 33,2 & 2.98 & 33,1 \\
\hline No será necesaria la asistencia presencial & 1.68 & 5,4 & 2.41 & 19,4 \\
\hline Descenderá el número de estudiantes que irán a las tutorías & 2.89 & 33,2 & 3.12 & 34,5 \\
\hline Permitirá hacer consultas sin desplazamientos & 4.43 & 90,9 & 3.95 & 67,4 \\
\hline No será necesario asistir tanto a las tutorías & 2.80 & 25,9 & 3.09 & 34,1 \\
\hline $\begin{array}{l}\text { Permitirá acceder a la información superando las barreras de espacio } \\
\text { y tiempo }\end{array}$ & 4.23 & 82,5 & 3.76 & 59,6 \\
\hline Se podrá compatibilizar los estudios con otras tareas u obligaciones & 3.41 & 47,2 & 3.43 & 46,2 \\
\hline
\end{tabular}

Tabla 30.7. Consecuencias relacionadas con la asistencia presencial

Por último, en la valoración de profesores y estudiantes sobre el uso del Campus Virtual como apoyo a las asignaturas presenciales y, a partir de su propia experiencia en el uso de la misma, a través del ítem el Campus Virtual de la ULPGC cubre de manera satisfactoria mis necesidades como apoyo a las asignaturas que imparto/ curso de manera presencial, ha obtenido una media muy superior al punto medio y cercana a 4 en el caso de los profesores y algo inferior por parte de los estudiantes (Ver Tabla 30.8).

\begin{tabular}{|l|l|l|l|l|l|}
\hline & \multicolumn{2}{|l|}{ Profesores } & \multicolumn{2}{l|}{ Estudiantes } \\
\cline { 2 - 6 } & $\bar{X}$ & $\mathbf{4 + 5}$ & $\bar{X}$ & $\mathbf{4 + 5}$ \\
\hline $\begin{array}{l}\text { EI Campus Virtual de la ULPGC cubre de manera satisfactoria mis necesidades } \\
\text { como apoyo a las asignaturas que imparto/curso de manera presencial }\end{array}$ & 3.94 & 81,4 & 3.57 & 52,7 \\
\hline
\end{tabular}

Tabla 3o.8. Valoración del Campus Virtual de la ULPGC

\section{TRABAJO COLABORATIVO}

Paralelo al uso de la plataforma de teleformación como apoyo a la enseñanza presencial comenzó el uso no docente de la misma. En este caso se trataba de utilizarla como un entorno colaborativo que permite a grupos "reunirse" de manera virtual y compartir un espacio común con documentos, informaciones varias, etc. Este servicio está abierto a todo el profesorado y el personal de administración y servicios de la ULPGC pudiendo participar también miembros ajenos a la ULPGC (Castro y 
Rodríguez, 2005). Los grupos de trabajo colaborativo que utilizan la plataforma de teleformación no ha dejado de crecer año tras año. En estos momentos más de 133 grupos participan activamente. En la tabla siguiente podemos ver un resumen de los mismos (Ver Tabla 31).

\begin{tabular}{|l|l|}
\hline ÁREA DE TRABAJO COLABORATIVO & No DE GRUPOS \\
\hline Docencia & 35 \\
\hline Investigación, desarrollo e innovación & 61 \\
\hline Gestión y servicios a la comunidad universitaria & 28 \\
\hline Impacto social y servicios a la sociedad & 9 \\
\hline
\end{tabular}

Tabla 31. Distribución por áreas de los grupos que utilizan el entono virtual de trabajo

\section{CONCLUSIONES Y PERSPECTIVAS DE FUTURO}

El crecimiento del Campus Virtual de la ULPGC, tanto por el número de usuarios como por sus funcionalidades, no para de crecer curso tras curso. Las previsiones apuntan a un nuevo incremento de profesores y estudiantes activos a finales de este curso. Para el próximo, ya está prevista la incorporación de nuevos títulos de grado y de posgrado en modalidad no presencial a través de teleformación.

Ante el incesante uso de los recursos TIC de tipo lúdico-social disponibles (cafetería, "busca y encuentra", etc.) se está ultimando los preparativos para una cuarta instalación dedicada a las delegaciones y asociaciones de estudiantes, aulas culturales, gestión administrativa diaria de centros y departamentos, etc. Se trata de una instalación con un fin más social y de relaciones entre los miembros de la comunidad universitaria dado que las ya existentes son de tipo más formal.

\section{NOTAS}

$1 \quad$ Ciencias Experimentales y de la Salud

2 Ciencias Sociales y Jurídicas

3 Enseñanzas Técnicas

4 Humanidades 


\section{REFERENCIAS BIBLIOGRÁFICAS}

Castro Sánchez, J. J. (2007). El modelo de teleformación de la Universidad de Las Palmas de Gran Canaria. Las Palmas de Gran Canaria: Servicio de Publicaciones de la Universidad de Las Palmas de Gran Canaria.

Castro Sánchez, J. J. (Coor.) (2006). Docencia universitaria a través de entornos virtuales de enseñanza-aprendizaje. Las Palmas de Gran Canaria: Servicio de Publicaciones de la Universidad de Las Palmas de Gran Canaria.

Castro Sánchez, J. J.; Rodríguez Díaz, J. M. (Coor.) (2005). Las tecnologías de la
Información y comunicación (TIC) en la docencia universitaria. Las Palmas de Gran Canaria: Servicio de Publicaciones de la Universidad de Las Palmas de Gran Canaria.

Castro Sánchez, J. J.; Chirino Alemán, E. (2004).Lastecnologíasdelainformación y comunicación (TIC) como apoyo a la enseñanza presencial en la Universidad de las Palmas de Gran Canaria. Las Palmas de Gran Canaria: Servicio de Publicaciones de la Universidad de Las Palmas de Gran Canaria.

\section{PERFIL ACADÉMICO Y PROFESIONAL DEL AUTOR}

José Juan Castro Sánchez. Licenciado y doctor en Psicología, profesor titular de la Universidad de Las Palmas de Gran Canaria. Desde 1998 dirige el programa de doctorado: Formación del Profesorado que se imparte a través de Teleformación. Es el responsable del Campus Virtual de esta universidad desde su creación hasta la actualidad. Ha dirigido 6 tesis doctorales y tiene varias publicaciones relacionadas con el uso de las TIC en la enseñanza.

E-mail: jcastro@dps.ulpgc.es

DIRECCIÓN DEL AUTOR:

Departamento de Psicología y Sociología.

Facultad de Formación del Profesorado.

Santa Juana de Arco, 1

CP: 35004

Las Palmas de Gran Canaria.

España

Fechas de recepción del artículo: 17/10/07

Fechas aceptación del artículo: 19/12/07 